\title{
Le renoncement comme forme de choix modal : l'abandon de l'usage du métro par les personnes vieillissantes
}

\author{
Renunciation as a form of modal shift: \\ how ageing people are abandoning the use of the metro
}

\author{
Marion Tillous \\ Reçu le 19 juillet 2013, accepté le 6 mars 2014 \\ (C) IFSTTAR et Éditions NecPlus 2014
}

Résumé Le renoncement des personnes vieillissantes à l'usage du métro est un phénomène de grande ampleur qui se traduit par un usage accru du bus et de la voiture en tant que passager, mais également par un renoncement à toute mobilité. La compréhension de ce phénomène comporte donc le double enjeu de réduction de la part accordée à l'automobile chez les personnes âgées et d'amélioration de leur autonomie. La recherche proposée porte sur les cas de Paris et de Montréal, et procède par entretiens qualitatifs. Elle a pour principal résultat de démontrer le rôle central dans le renoncement, en plus des critères d'ordre fonctionnels (physiques et cognitifs), de la modification du rapport à l'environnement socio-spatial. La dimension sociale du renoncement se traduit ainsi par : le stigmate, la peur de ne pas trouver d'aide, la reconfiguration de la demande de sociabilité. La dimension spatiale/géographique se traduit par une difficulté grandissante à l'attachement territorial corrélative de l'émergence d'un sentiment d'étrangéité. Ces résultats invitent à renouveler la question de l'explication du choix modal à partir de la notion du renoncement, ce qui se traduit par : une nouvelle manière d'aborder la notion de choix, «par exclusion »; une façon de comprendre les limites du rôle de l'habitude dans le choix modal ; un argument en faveur de l'approche méthodologique qualitative et située (expérience en situation, événement in situ, attachement à un espace).

Mots clés transports urbains · choix modal · vieillissement $\cdot$ méthodes qualitatives $\cdot$ interactions $\cdot$ déprise

L'auteure remercie Théo Fort-Jacques, docteur en GéographieAménagement, pour les nombreux échanges à partir desquels cet article a pu voir le jour. Cette recherche a bénéficié du soutien de l'Action Interdisciplinaire de Recherche « Longévité et Vieillissement » du CNRS pour la réalisation du terrain montréalais.

Marion Tillous $(\bowtie)$

Département de Géographie - UFR TES - Université Paris 8

Vincennes Saint-Denis 2, rue de la liberté - 93526 Saint-Denis Cedex e-mail : marion.tilllous@univ-paris8.fr

\begin{abstract}
Renunciation of the metro by the elderly is a widespread phenomenon that leads to a higher use by the elderly of buses and cars as passengers, but also to the abandonment of mobility itself. The importance of understanding this phenomenon is two-fold: to decrease the modal share of private car use among aging people, and to guarantee their autonomy. The research presented here is based on qualitative research in the form of interviews using Paris and Montreal as case studies. It demonstrates the crucial role in the metro renunciation played - aside from functional (physical and cognitive) factors - by the changes in relations to the socio-spatial environment that occur during aging. The social dimension here manifests in: social stigma, fear of not finding any help in case of distress, and change in social needs. The spatial/geographical dimension manifests in an increasing difficulty to form attachment to a space as a form of territory, originating from the feeling of being an outsider. These results lead us to rethink the issue of modal choice using this notion of renunciation. Specifically, it provides: a new way of defining the notion of modal choice as a form of default choice; a tool to understand the limits of habit as an explanation for modal choice; an argument in favor of qualitative methods that focus on situations (experience in situation, in situ events, territorial attachment).
\end{abstract}

Keywords urban transport - modal choice - aging · qualitative methods · interactions · "déprise"

\section{Introduction}

Conséquence du baby-boom des années 1950 et de l'amélioration des conditions de vie, la population des pays occidentaux vieillit et reste de plus en plus longtemps en bonne santé. L'Insee projette ainsi que la population des plus de 60 ans passera de $21,5 \%$ en 2007 à $31 \%$ en 2040 : plus d'une personne sur trois sera âgée de 60 ans ou plus au milieu du XXI ${ }^{\mathrm{e}}$ siècle. À l'horizon 2060, c'est une 
augmentation de $80 \%$ de la part des plus de 60 ans dans la population totale qui est annoncée par l'Insee [1].

La mobilité des personnes âgées et les reconfigurations qu'impose le vieillissement représentent de ce fait une question vive et sont depuis longtemps étudiées. Mais ce domaine de recherche est dominé par des approches médicales qui se focalisent sur la conduite automobile [2] : l'enjeu étant de réduire l'insécurité routière liée aux déficiences physiques (vue, sommeil) et cognitives (mémoire, réflexe) qui résultent de la sénescence. Les autres modes de transport, collectifs ou non mécanisés, ne sont généralement appréhendés qu'en tant qu'alternative à l'automobile [3], et sont donc envisagés de manière intégrée, sans distinction modale, dans le traitement statistique des enquêtes sur les déplacements $(c f$. $\S$ «Ampleur du phénomène »).

Les enjeux propres aux conséquences du vieillissement sur la mobilité en transports collectifs sont moins clairement perçus car ils concernent l'autonomie de la personne plus que sa sécurité. La perte d'autonomie liée à la cessation de l'usage des modes de transports collectifs par les personnes vieillissantes est moins perçue comme un problème mais comme une conséquence nécessaire d'un processus irréversible, le vieillissement. Nous verrons ( $c f$. $\S$ «Enjeux ») qu'une telle approche relève d'une conception dépassée du vieillissement, celle du « désengagement » par opposition à la « déprise », et qu'il y a un double enjeu à la remettre en cause dans le domaine des déplacements : celui de l'autonomie de la personne, mais également celui de la diminution de la part modale de l'automobile qui concerne, elle, l'ensemble de la société.

Transposée au champ de la mobilité, l'approche du vieillissement comme " déprise » offre un cadre théorique déterminant pour comprendre l'enjeu d'une analyse des reconfigurations du choix modal au grand âge, et en particulier du renoncement à l'usage des modes urbains lourds, et pour proposer une explication à ces reconfigurations (cf. $\S$ « Résultats »). Nous verrons en outre que l'entrée par le renoncement, donc par le choix modal par défaut pris postérieurement à la mise en place d'une routine, ainsi que les résultats obtenus à propos du grand âge permettent de repenser la façon dont le choix modal est abordé aujourd'hui, toutes catégories d'âge confondues, et de proposer de nouvelles méthodes d'enquête ( $c f$. $\S$ «Discussion »). La compréhension du phénomène du renoncement à l'usage du métro s'appuiera sur l'étude des cas de Montréal et de Paris.

Avant d'aborder la démonstration, une précision s'impose : qu'entendons-nous par «métro »? Le métro est un réseau qui tire sa spécificité de sa fermeture, de sa quasi-indépendance à l'égard du tissu urbain. La dimension souterraine renforce cette séparation, mais elle n'est pas ce qui distingue le métro des autres modes, puisqu'il arrive fréquemment que des lignes soient en partie aériennes ; mais elles restent coupées de l'espace public urbain par les barrières de contrôle. Ces barrières permettent en revanche que le système soit organisé selon des principes gestionnaires très spécifiques, que le sociologue pragmatiste Isaac Joseph ${ }^{1}$ a mis en évidence lors d'une recherche croisée sur les modes bus et métro [4,5] : la rapidité, la lisibilité, la régularité et l'uniformité du service rendu. Nous associerons donc le métro aux autres modes ferrés urbains lorsque ceux-ci sont enclos (RER, en particulier), mais pas aux modes ferrés aériens, tels que les tramways et lignes de banlieue, ni aux autres modes de transports collectifs comme les bus.

\section{Position du problème}

\subsection{Ampleur du phénomène : une faible visibilité dans les traitements statistiques}

La mesure de l'évolution de la répartition modale au cours $\mathrm{du}$ vieillissement est assez difficile dans la mesure où cette question n'est pas encore constituée comme question de recherche. Les données qui existent sont ainsi, pour la plupart, discriminées soit en fonction de l'âge, et donnent alors une répartition modale qui ne distingue par les transports collectifs entre eux, soit en fonction du mode, et l'âge cesse d'être une variable discriminante, ou seulement dans ses grandes divisions (enfance, jeunesse, âge adulte, vieillesse).

En France, les données issues des Enquêtes ménages déplacements (EMD) par exemple ont pu donner lieu à des analyses précises de la mobilité des personnes âgées [6]. Si les auteurs observent une plus grande fréquentation des transports collectifs dans les villes bénéficiant d'un Transport en commun en site propre [6, p. 28], cela ne les conduit pas pour autant à proposer une analyse de la répartition modale selon l'âge distinguant entre eux les transports en commun. Cette absence n'est pas un oubli mais signifie que l'analyse de la répartition modale visait à répondre avant tout à la question des alternatives existant à l'automobile au cours du vieillissement. Les données issues des opérateurs, plus précises sur les modes, ne permettent que rarement de connaître l'âge des voyageurs effectifs, tous titres de transport confondus. Surtout, elles ne permettent pas de placer ces données relatives aux déplacements en métro dans le contexte de la mobilité de la personne, tous modes confondus. Lorsqu'elles existent, les enquêtes sur un public spécifique réalisées par les opérateurs intègrent les personnes vieillissantes aux personnes à mobilité réduite,

\footnotetext{
${ }^{1}$ Qui fut chercheur associé à la Mission Prospective de la RATP de 1985 à son décès en 2004.
} 
celles-ci étant discriminées par handicap fonctionnel et non selon des critères sociodémographiques.

À l'international, le même constat peut être porté $[7,8]$. À Montréal, la seconde ville choisie comme terrain de recherche ( $c f . \S$ «Enquête : étude de cas et méthodologie»), l'enquête Origine-Destination, équivalent québécois des EMD, a ainsi donné lieu à traitement très précis de la distribution spatiale des déplacements [9], mais n'a pas exploité les informations prélevées en termes d'âge autrement que pour constater que «le vieillissement de la population se poursuit $\gg[10$, p. 19] .

Les données issues de l'Enquête globale transport (Ile-de-France) la plus récente ont pourtant donné lieu à un traitement plus détaillé. Si la répartition modale des déplacements des plus de 60 ans ne distingue toujours pas les transports collectifs entre eux, un tableau spécifique précisant la part modale du bus dans les déplacements réalisés en transports collectifs a fait son apparition [11, p. 4].

Les données présentées dans ce tableau indiquent que la part du bus dans les transports collectifs est bien supérieure pour les retraités ( $40 \%$ ) que pour l'ensemble des franciliens (25\%). Elles permettent de vérifier également que cette part augmente constamment à partir de 61 ans : pour la classe d'âge la plus élevée (81 ans et plus), la part du bus dans les transports collectifs atteint les $56 \%$, alors qu'elle est de $29 \%$ seulement pour les 61-65 ans. Rapporté à un nombre moyen de déplacements par jour en forte diminution selon l'âge ${ }^{2}$, et à un nombre absolu de déplacements en transports collectifs qui n'augmente pas et tend même à décroître à partir de 75 ans, ce document apporte la preuve que le nombre de déplacements réalisés en transports collectifs hors bus (métro, RER, tramway, donc) diminue avec l'âge.

Un traitement spécifique réalisé par Yves Bussière et Jean-Pierre Thouez [12, cité par 13, p. 21] pour le cas de l'Île de Montréal à partir de données de 1998, permet d'affirmer que la diminution relative de l'usage des modes collectifs ferrés avec l'âge existe également dans cette métropole et ce, depuis longtemps. La part modale des modes ferrés (train, métro) apparaît en effet en diminution avec l'âge puisqu'elle passe de $14,2 \%$ pour les moins de 75 ans à $6,8 \%$ pour les 75 ans et plus. Dans la mesure où le nombre moyen de déplacements est lui-même en diminution avec l'avancée en âge (2,16 déplacements pour les 65-74 ans et 1,41 pour les plus de 75 ans en 2006 [13, p. 17]), le nombre absolu de déplacements en métro et train diminue avec l'âge à Montréal comme à Paris. La part modale du bus, elle, augmente avec l'âge ${ }^{3}$.

Le renoncement à l'usage du métro au cours du vieillissement apparaît donc, à la lecture des quelques

\footnotetext{
2 4,08 en moyenne pour les 61-70 ans, puis en diminution progressive jusqu'à 2,55 pour les plus de 80 ans

${ }^{3} 8,7 \%$ pour les moins de 75 ans contre $12,9 \%$ pour les 75 ans et plus
}

données quantitatives disponibles, de grande ampleur. Manquent pourtant pour asseoir ce constat un traitement plus systématique des données portant sur la part modale en fonction de l'avancée en âge, ainsi que la production de données longitudinales sur l'évolution du choix modal au cours du vieillissement. Les rares enquêtes longitudinales existant aujourd'hui se focalisent en effet sur les territoires dépendants à l'automobile comme les périphéries pavillonnaires [14] donc non desservis par les réseaux ferrés urbains. Pourtant, il existe un enjeu fort à mieux connaitre ce phénomène.

\subsection{Enjeux de l'étude du renoncement}

Pour évaluer au plus juste les conséquences du renoncement à l'usage du métro, il faut se demander comment les personnes vieillissantes réalisent les déplacements auparavant effectués en métro une fois qu'elles ont renoncé à ce mode. Le traitement des données de l'EGT 2009-11 présenté plus haut semble suggérer que le transfert modal se fait au profit $\mathrm{du}$ bus. Mais on constate que ce renoncement se fait aussi en faveur de la voiture particulière (en tant que passager - cf. 11, p. $4^{4}$ ) ou peut se traduire par une adaptation du déplacement (destination ou motif) pour permettre de le réaliser à pied. Cette adaptation peut signifier renoncement au déplacement lui-même.

La question du renoncement à l'usage du métro présente donc un double enjeu :

1. pour penser une mobilité durable, s'intéresser au report modal de la voiture vers les modes alternatifs ne suffit pas, encore faut-il éviter que le report inverse, des transports collectifs vers la voiture ne se produise. Le report modal est un levier central des politiques de mobilité urbaine pour réduire les pollutions aériennes et sonores, diminuer l'insécurité routière, ou encore favoriser des formes durables d'urbanité ; les collectivités territoriales y consacrent une part considérable de leurs moyens. Il semble absurde de ne pas, en parallèle, chercher à conserver les usagers qui sont déjà acquis à l'usage des transports collectifs, action qui semble moins lourde et moins coûteuse.

2. le deuxième enjeu concerne l'autonomie de la personne elle-même. L'isolement des personnes âgées est un problème qui s'accentue à mesure que la population

\footnotetext{
${ }^{4}$ La part des passagers parmi les usagers de la voiture augmente très nettement passé 75 ans. Même si l'on tient compte de la diminution de l'usage général (conducteur et passager) de la voiture au cours de l'âge, la part des passagers de l'automobile rapportée à l'ensemble des déplacements tous modes confondus est elle-même en augmentation passés 75 ans $(6,8 \%$ entre 71 et 75 ans, $8,25 \%$ entre 76 et 80 ans, $8,32 \%$ après 80 ans).
} 
vieillit [15]. Les acteurs de la mobilité urbaine ont un rôle central à jouer pour assurer aux personnes vieillissantes de conserver une palette de choix étendue pour accéder aux ressources urbaines, y compris aux ressources sociales. Cela suppose d'envisager l'isolement des personnes vieillissantes non pas comme un processus inéluctable mais comme le résultat d'une impossible reconfiguration des stratégies de mobilité au cours du vieillissement du fait d'un environnement socio-spatial inadapté.

Tout se passe en effet comme s'il persistait ainsi dans le champ de la mobilité une approche théorique remise en cause dans la plupart des autres domaines qui est celle de la théorie du «désengagement» [16]. Celle-ci envisage le vieillissement normal comme un désengagement progressif et irréversible de la personne qui vieillit à l'égard du système social dans lequel elle s'inscrit. Ce désengagement serait réciproque, le système de mise à la retraite venant appuyer un retrait engagé par la personne elle-même. Il aurait pour fonction de limiter la vulnérabilité des personnes vieillissantes à l'égard de l'idée de leur finitude (le désengagement comme " passage en douceur d'une vie, qui a été longue, à la mort inévitable »), mais également celle du système qui risquerait de se trouver déstabilisé par la disparition soudaine de personnes occupant des fonctions importantes. En somme, la diminution de la mobilité dans l'espace et dans le temps résulterait de ce processus universel et irréversible.

Il semble donc nécessaire d'inscrire l'analyse de la mobilité des personnes vieillissantes dans le champ théorique de la « déprise » $[17,18]$, qui oppose au « désengagement » l'idée d'une reconfiguration des activités au cours du vieillissement faite d'adaptations et de négociations avec l'univers physique comme avec le système social. Cette approche est fondamentalement relationnelle : elle suppose qu'au cours de l'avancée en âge et de l'apparition croissante de difficultés fonctionnelles, les personnes vont reconfigurer leurs activités en fonction de (donc en relation avec) leur environnement socio-spatial. Car, en effet, cette déprise est tout autant spatiale que sociale : les mêmes auteurs [19] ont montré qu'au cours du vieillissement, les personnes étaient amenées à reconfigurer leurs espaces de pratiques quotidiennes. Ils ont en particulier constaté que « la fréquentation ou la non-fréquentation des lieux urbains par les vieilles personnes n'emprunte que superficiellement aux critères de stricte proximité ou facilité d'accès. Certains espaces éloignés, d'accès a priori plus long et plus complexe, peuvent être considérés comme plus accessibles que l'espace contigu au logement ».

Cette approche théorique du vieillissement comme déprise est utile, non seulement pour comprendre les enjeux à l'étude du renoncement, mais également pour apporter un éclairage complémentaire à la réponse apportée jusqu'à ce jour par les opérateurs, à savoir la réponse ergonomique.

\subsection{Réponse opérationnelle : une amélioration de l'ergonomie}

Le renoncement à l'usage du métro résulte du fait qu'à mesure du vieillissement, la fréquentation des espaces de réseau ferré induit une pénibilité supérieure au bénéfice qui en est retiré dans un contexte de programme d'activité libéré de contraintes professionnelles et scolaires. Cette pénibilité a été prise en compte par les autorités et opérateurs de transport public qui ont envisagé la personne vieillissante sous l'angle de ses déficiences physiques et cognitives et ont cherché à imaginer des environnements adaptés à ses besoins. Les cas de Paris et de Montréal montrent que deux visions se sont opposées, qui relèvent toutefois de cette approche ergonomique de la personne vieillissante.

Dans la région métropolitaine de Montréal, l'opérateur STM a adopté dès le début des années 1980 le parti de proposer une offre de transport spécialisée pour les personnes porteuses d'un handicap fonctionnel permanent ${ }^{5}$ sous la forme de bus "adaptés » à leurs besoins. Plus récemment, des minibus plus spécifiquement destinés aux personnes âgées ont été introduits sous l'appellation Navettes Or ( $c f$. $\S$ « Choix des cas d'étude : Paris et Montréal »). La mise en accessibilité du réseau de métro, si elle est aujourd'hui reconnue comme nécessaire, n'est toujours pas une priorité budgétaire : en 2003, le Comité sur l'accessibilité du métro de Montréal constate ainsi que « À l'heure actuelle, le métro de Montréal est l'un des derniers métros du monde à n'être aucunement accessible aux personnes en fauteuil roulant » [20, chap.3]. Sur les six stations identifiées par le comité comme à rendre accessibles en priorité, quatre seulement sont, en 2013, équipées d'ascenseurs, auxquelles s'ajoutent les trois nouvelles stations du nord-est de la ligne orange, équipées d'emblée. Les coûts très élevés de la mise en accessibilité de stations existantes expliquent ces réticences, puisque la STM estimait que la réalisation des travaux dans les 6 stations identifiées par le comité coûterait entre $300000 \$$ et $400000 \$[20$, p. 22].

En Ile-de-France, autorités et opérateurs se sont paradoxalement engagés plus tardivement dans une démarche visant à assurer l'accès des personnes à mobilité réduite aux ressources urbaines. Leurs efforts se sont donc concentrés sur une accessibilité de la deuxième heure, «intégrée »

\footnotetext{
${ }^{5}$ Ce handicap, physique ou mental, doit être attesté par une autorité médicale, et correspondre à l'un des handicaps listé par l'opérateur. Il ne s'agit donc que des handicaps lourds et permanents, et non des handicaps circonstanciels tels que le portage de bagages, ou de handicaps légers tels que la fatigue, les vertiges ou une mauvaise vue.
} 
et non plus « spécifique », c'est-à-dire sous la forme d'aménagements intégrés au réseau commun et non plus sous la forme de réseaux spécifiques [21]. Cette démarche présente l'avantage de s'adresser à l'ensemble des personnes à mobilité réduite, y compris les personnes porteuses de handicaps légers ou circonstanciels. Depuis les années 1980 et surtout 1990, les espaces de mobilité ont ainsi été pensés et aménagés dans un souci d'ergonomie et d'accessibilité, les personnes âgées étant assimilées à une catégorie spécifique de personnes à mobilité réduite.

Cette évolution s'inscrit dans le cadre d'une rupture plus générale dans la conception du réseau : durant cette période, le « réseau-tuyau » aménagé pour le flux est remis en cause comme référence opérationnelle au profit d'une attention portée aux voyageurs comme des individus ayant des besoins et des envies propres [22]. Cette rupture est la conséquence d'une modification de l'organisation sociale de l'opérateur : avec l'automatisation du système de transport, un grand nombre de postes auparavant en contact avec le public disparaissent. La relation de service doit être repensée et intégrer l'espace : les agents ne sont plus les seuls dépositaires de la charge d'accompagnement des voyageurs ( $c f$. travaux de l'Unité prospective de la RATP et notamment : [23]). L'accessibilité des espaces devient alors une part de la relation de service [21, chap.7]. Des efforts de conception des espaces du métro sont alors faits pour permettre une meilleure prise en compte des spécificités de chacun ; le terme d'accessibilité, comme facilité d'accès physique et cognitif devient central. Isaac Joseph parle ainsi à propos des gares d'un « monde d'intelligence distribuée » $[24$, p. 13] et de la nécessité pour les espaces de se donner à lire comme des « modes d'emplois » [24, p. 12].

Mais deux dimensions de l'expérience du déplacement sont escamotées au cours de cette conversion [25]. Dans une conception individualisée du voyageur disparaît tout d'abord la dimension sociale de son expérience : or, à quoi bon mettre en place des ascenseurs par exemple si leur usage est stigmatisant? D'autre part, l'approche ergonomique tend à comprendre l'expérience du déplacement comme la mise en œuvre d'une interaction homme-machine : elle laisse de côté la dimension géographique de la pratique d'un espace. Celle-ci se distingue de la pratique de l'outil parce qu'elle est sujette à un attachement territorial : une personne qui pratique pour la première fois un espace ne se sent pas uniquement novice ou maladroite, mais « étrangère » à un territoire.

Interactions sociales et attachements spatiaux entrent dans le cadre des relations qui engagent la déprise et ses adaptations situées au cours du vieillissement. C'est bien pourquoi ces deux dimensions (sociale et géographique) de l'expérience du voyageur jouent un rôle dans le renoncement du métro au cours du vieillissement, ce que nous avons pu mettre en évidence à l'occasion de notre travail doctoral [26, et plus précisément : 27] et asseoir au cours d'une recherche complémentaire.

\section{Enquête : étude de cas et méthodologie}

\subsection{Choix des cas d'étude : Paris et Montréal}

À l'origine de cette recherche se trouve notre travail doctoral, réalisé entre 2005 et 2009 , et portant sur le réseau de transport de l'agglomération parisienne. Quatre pôles d'échange avaient alors été choisis comme études de cas (Gallieni, Massy-Palaiseau, Pont de Sèvres et Villejuif Louis-Aragon) mais les expériences de voyageurs recueillies ont porté sur l'ensemble des espaces de réseau fréquentés. Lors de cette recherche initiale, des signaux faibles ont été identifiés au sein des récits de personnes vieillissantes ayant renoncé au métro. Des entretiens avec des personnes plus jeunes ayant elles aussi renoncé au métro ont permis de penser qu'il existait une spécificité du renoncement au cours du vieillissement. Pour vérifier cela et asseoir nos conclusions, nous avons souhaité mettre en place une recherche comparative dans une autre métropole d'échelle mondiale. Celle-ci s'est déroulée au cours de l'année 2011.

Le cas de Montréal présentait l'intérêt de posséder un dispositif d'accompagnement des personnes à mobilité réduite au sein des espaces communs de mobilité. À Paris, ce service est payant et il est assuré par l'association les Compagnons du Voyage. À Montréal, la STM a intégré ce service d'accompagnement à ses missions et l'a rendu à la fois plus lisible et plus institutionnel : toute personne qui souhaite se faire accompagner peut en faire la demande au moment de son déplacement au guichet de sa station de départ. Un accompagnateur sera alors dépêché pour l'accompagner du guichet de départ au guichet d'arrivée. Une « carte d'accompagnement » a également été mise en place pour que les personnes puissent se faire accompagner dès le début de leur déplacement par un proche : celui-ci ne paye alors pas son déplacement. La carte est gratuite et délivrée à toute personne reconnue par une instance médicale agréée comme "vivant avec une déficience intellectuelle ou visuelle ». La possibilité d'être accompagné au cours de son déplacement nous a semblé être un révélateur intéressant des freins d'ordre sociaux à l'usage du métro au grand âge.

Le fait que la ville de Montréal soit pour grande partie francophone rendait possible la mise en œuvre d'une analyse sémantique des entretiens réalisés au même titre que pour le cas parisien. Même cette proximité linguistique n'a pas évité la nécessité d'une table d'équivalence pour éviter les confusions et approximations. Par exemple, l'expression « chez nous » employée au Québec témoigne moins d'une appropriation collective d'un espace (précisément l'objet 
que nous cherchions à identifier) que d'un attachement avant tout personnel. Il est plus juste de la considérer comme équivalente au « chez soi » français.

\subsection{Offre de mobilité pour les personnes vieillissantes dans les deux cas d'étude}

Ces dispositifs d'accompagnement se font dans les réseaux de transports (en) communs, donc les réseaux de bus et de métro. La métropole montréalaise, peuplée de 2,3 millions d'habitants $^{6}$ sur une surface de $746 \mathrm{~km}^{2}$, est plus petite que la métropole parisienne qui compte cinq fois plus $\mathrm{d}^{\prime}$ habitants sur une surface quatre fois plus grande ${ }^{7}$. Son centre-ville, l'Île de Montréal, est moins dense que celui de Paris. La ville de Montréal est d'abord une ville aménagée pour une desserte automobile. Si son réseau de bus est conséquent, son réseau de métro est bien plus restreint que celui de Paris : il comporte quatre lignes de taille inégale quand Paris en comprend 16. Si la ligne orange (de Côte-Vertu à Montmorency) dessert deux axes nord-sud, l'axe principal est-ouest du centre des affaires et les principaux centres des villes francophones et anglophones, la ligne jaune (de Berri-UQAM à Longueuil) ne comporte que trois stations. Le réseau de métro montréalais, mis en service à partir de 1966, est renforcé par un réseau de trains de banlieue qui sillonne l'agglomération et assure le lien entre l'Île de Montréal et les rives du Saint-Laurent.

Il a déjà été indiqué que la mise en accessibilité du réseau de métro de Montréal avait été entreprise plus tardivement qu'à Paris. Sept stations du réseau seulement disposent d'un ascenseur, trois d'entre elles étant des stations construites récemment (2007) et non des réaménagements, qui sont extrêmement coûteux. Outre les ascenseurs, le remplacement des portes battantes très lourdes et l'implantation d'appuis ischiatiques $^{8}$ ont été entrepris, mais restent confidentiels. Pourtant, le métro de Montréal conserve parmi les actifs la réputation d'être réservé aux écoliers/étudiants et aux personnes âgées, c'est-à-dire aux captifs des transports collectifs. La mise en accessibilité du réseau de bus a été entreprise à Paris comme à Montréal à la fin des années $1990^{9}$. Elle s'est traduite par le remplacement du parc par des bus à planchers surbaissés avec rampes télescopiques, et par la surélévation des arrêts. Elle concerne maintenant l'ensemble du réseau.

Des réseaux spécifiquement destinés aux personnes vieillissantes comme personnes à mobilité réduite ont

\footnotetext{
${ }^{6}$ Selon le recensement 2011 pour les municipalités de Montréal et de Laval. Source : Institut de la statistique du Québec.

${ }^{7}$ Selon le recensement 2009 pour l'unité urbaine de Paris : 10,5 millions d'habitants sur une surface de $2845 \mathrm{~km}^{2}$. Source : Insee.

${ }^{8}$ Bancs « assis-debout » qui permettent de se reposer sans s'asseoir.

${ }^{9} 1997$ pour la STM et 1999 pour le Stif.
}

également été mis sur pied dans les deux agglomérations. Les principaux réseaux prennent la forme, à Paris comme à Montréal, de services de transport à la demande assurés par des minibus adaptés aux besoins spécifiques. Ils sont facilement accessibles depuis l'extérieur et peuvent par exemple accueillir les fauteuils roulants. Ils doivent être réservés la veille du déplacement et sont ouverts aux déplacements personnels comme professionnels. Le service de « transport adapté » montréalais a été mis en place au sein de l'opérateur public de transport en 1980 par intégration d'un service privé lancé dès 1973. Ce dispositif est donc bien plus ancien que son équivalent parisien nommé PAM (Paris accompagnement mobilité) et lancé en 2003. Il est accessible au même tarif que les autres modes collectifs à Montréal, pour un prix plus élevé à Paris. Les usagers âgés de 65 ans et plus représentent $57 \%$ de la clientèle du transport adapté montréalais, les trois quarts étant âgés de 75 ans et plus.

La spécificité du réseau de Montréal réside dans la mise en place, à compter de 2008 , du service Navette Or, des lignes de bus reliant à heures fixes et non à la demande les quartiers de résidence des personnes âgées aux centres commerciaux, médicaux et communautaires. La dizaine de lignes concernées fonctionne 2 à 3 jours par semaine en dehors des heures de pointe, c'est-à-dire entre $10 \mathrm{~h}$ et $15 \mathrm{~h}$ environ. Ce service est assuré par des minibus de 20 places non-accessibles aux fauteuils.

\subsection{Méthodologie d'enquête}

La recherche initiale à l'origine de ce travail est, rappelons-le, une recherche doctorale portant sur les déterminants de l'aisance au cours du déplacement urbain sans distinction d'âge. Trente-six personnes ont alors été rencontrées ; quatre d'entre elles étaient âgées de plus de 60 ans dont deux de plus de 75 ans ${ }^{10}$. Trois entretiens ont également été conservés de cette recherche car ils concernaient des personnes de moins de 60 ans ayant renoncé à l'usage du métro ${ }^{11}$. Les grilles d'entretien portaient sur l'expérience du déplacement dans toutes ses dimensions : usages du pôle choisi comme étude de cas et de ses équipements, ambiance ressentie, représentations liées au pôle, place du pôle dans le programme d'activités quotidiennes, image du pôle au regard des autres stations fréquentées. La réalisation d'une carte mentale accompagnait la passation de l'entretien. L'ensemble des entretiens a été retranscrit par nous mot à mot.

\footnotetext{
${ }^{10}$ Ces personnes sont désignées par le sigle PA (Personne âgée) suivi d'un numéro. Leurs profils, ainsi que ceux des autres personnes interrogées, sont détaillés en annexe.

${ }^{11}$ Ces personnes sont désignées par le sigle JRP (Jeune ayant renoncé - Paris) suivi d'un numéro.
} 


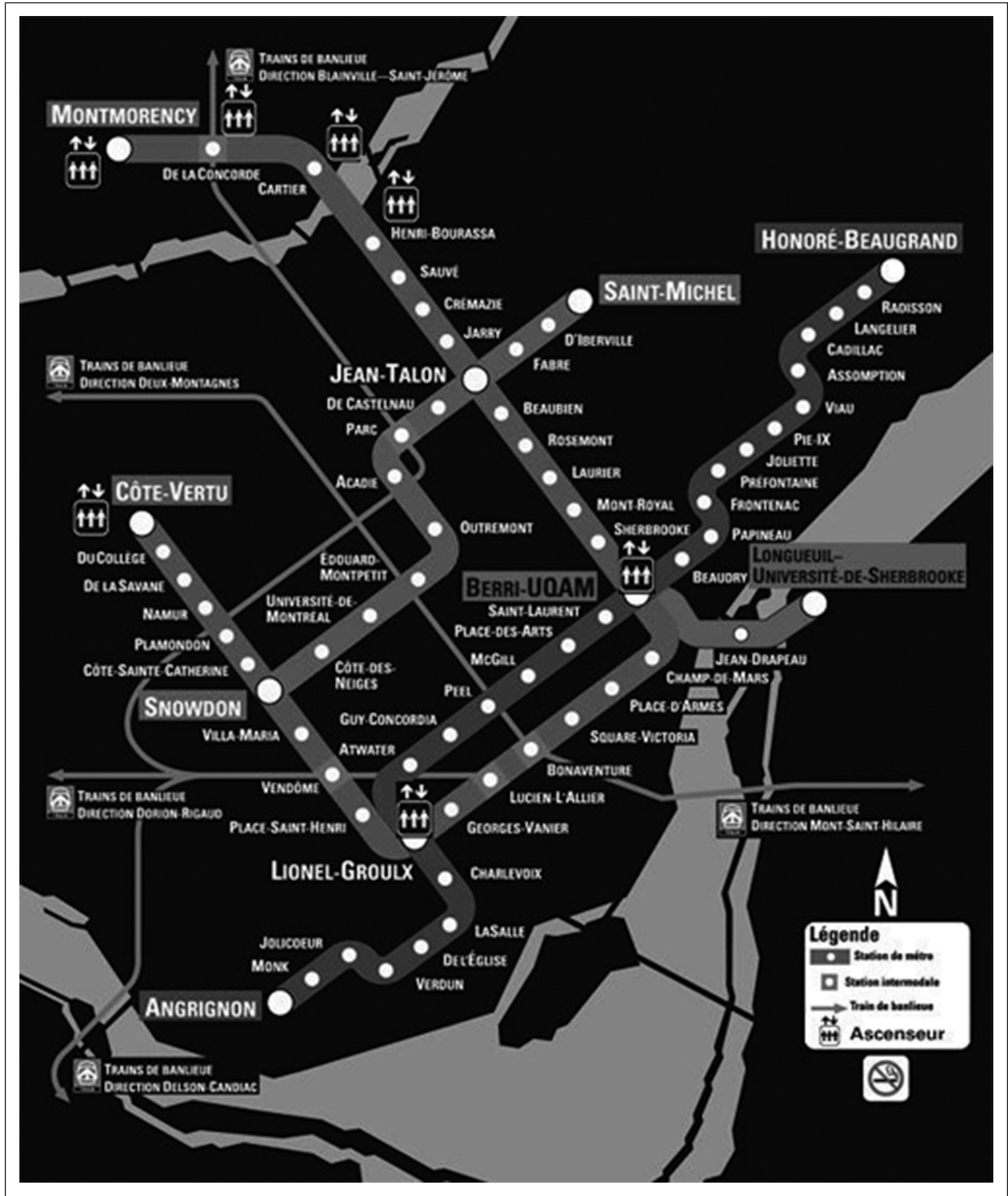

Fig. 1 Plan du réseau de métro de Montréal

Source : STM

La recherche complémentaire réalisée à Montréal s'est plus focalisée sur les personnes viellissantes et sur les dimensions sociale et géographique du déplacement. Seize personnes de plus de 65 ans ont été interrogées, sept d'entre elles ayant renoncé à l'usage du métro ${ }^{12}$. Quatre personnes

12 Le renoncement du métro est affirmé pour deux d'entre elles et de fait pour les cinq autres. Nous avons distingué ces deux profils en AR de moins de 50 ans ayant elles aussi renoncé à l'usage du métro ont été interrogées à titre de comparaison. Comme dans le cas parisien, les personnes interrogées ont été

(Aîné ayant renoncé) et ACR (Aîné en cours de renoncement). Les personnes vieillissantes n'ayant pas renoncé sont notées AU (Aînés utilisateurs). Quant aux plus jeunes ayant renoncé ils sont notés JRM (Jeune ayant renoncé - Montréal). 
pour beaucoup recrutées par connaissances interposées. Nous avons également fait appel à la médiation des centres communautaires et du service des Navettes Or pour étendre le nombre de personnes rencontrées. L'ensemble des entretiens a été retranscrit mot à mot par Véronique Vachet ${ }^{13}$.

La méthode employée se situe à l'intersection entre l'entretien semi-directif et l'entretien non directif. Une grille d'entretien a été constituée de façon à s'assurer de la progression de la conversation et du traitement exhaustif des thèmes. Elle servait également à rassurer la personne sur le rôle de l'enquêteur. Les questions n'étaient toutefois posées que si la personne n'y venait pas d'elle-même et si la relance à partir des termes employés par la personne elle-même n'était pas suffisante.

La grille d'entretien débutait par des questions portant sur le quartier de la personne de façon à la mettre à l'aise, et à la placer dans le récit et non dans les réponses factuelles attendues dans le cadre d'un questionnaire court. La personne était ensuite invitée à décrire ses activités quotidiennes et les déplacements qui y étaient associés, puis à préciser les modes de transport utilisés. Cette partie était destinée à identifier un éventuel renoncement au métro et à recueillir les valeurs associées par la personne à chacun des modes. La suite de la grille d'entretien se focalisait sur le métro : la personne était invitée à décrire étape par étape (entrée dans la station, descente jusqu'au hall d'accueil, achat de billet, etc.) sa pratique du métro, à évoquer les difficultés qu'elle pouvait y rencontrer, et à comparer son expérience avec celle du bus, de la rue ou de la ville souterraine ${ }^{14}$. La grille se focalisait ensuite sur la dimension sociale de l'expérience du métro (récit des interactions passées) puis, le cas échéant, sur le récit des conditions dans lesquelles la personne avait été conduite à renoncer à l'usage du métro et sur les conséquences de ce renoncement au quotidien. La grille se terminait par une question plus légère invitant les personnes les plus âgées de l'échantillon à raconter la construction et l'ouverture du métro et la façon dont cette ouverture a changé leur vie quotidienne.

\footnotetext{
${ }^{13} \mathrm{La}$ totalité des entretiens ainsi que la grille d'entretien sont disponibles sur demande auprès de l'auteure.

${ }^{14} \mathrm{La}$ ville souterraine est un important réseau de corridors reliant les étages inférieurs des immeubles du centre des affaires (dont le centre peut être situé à la Place Ville-Marie) de façon à permettre d'accéder à un grand nombre de commerces, de restaurants et d'autres services urbains depuis différentes stations de métro et parkings sans avoir à sortir dans la rue. Ce réseau est également relié aux immeubles de bureau. La ville souterraine est désormais appelée « ville intérieure » de façon à limiter la connotation d'enfermement et d'étouffement liée au souterrain. De fait, la moitié du réseau piéton se trouve en rez-de-chaussée ou à l'étage des immeubles reliés (Source : Observatoire de la ville intérieure). Dans la grille d'entretien, l'expression de « ville souterraine » a été conservée de façon à bien suggérer le parallèle avec le réseau de métro.
}

\section{Résultats : \\ le rôle des dimensions sociale et géographique dans le renoncement}

\subsection{La difficulté fonctionnelle (physique et cognitive) : une explication partielle du renoncement}

L'ensemble de résultats développés au cours de cette partie porte sur les raisons du renoncement à l'usage du métro des personnes vieillissantes. Nous verrons ensuite au cours de la « discussion » que ce travail permet d'améliorer la connaissance du choix modal de l'ensemble des personnes en déplacement, quel que soit leur âge.

Nous souhaitons montrer ici qu'interviennent dans le renoncement au métro des raisons d'ordre social et géographique. Il ne s'agit pas de dire que ces raisons sont les seules et qu'elles se substituent aux raisons physiques et cognitives. $\mathrm{Au}$ cours du vieillissement, la personne rencontre différents événements de santé qui se traduisent par des difficultés physiques (douleurs, fractures, arthrose, baisse des capacités) ou cognitives (mémoire, difficulté exécutive, difficulté attentionnelle) qui sont de nature à provoquer le renoncement. Mais si l'événement de santé est dans la plupart des cas à l'origine du renoncement, la difficulté fonctionnelle qui en résulte n'explique qu'en partie ce renoncement.

Il suffit pour s'en convaincre d'observer que dans de nombreux cas les obstacles physiques ou cognitifs qui paraissent insurmontables dans le métro sont perçus comme praticables dans d'autres contextes socio-spatiaux. La perception des escaliers est celle qui varie le plus selon les situations. À deux reprises, des personnes interrogées (PA_01 et ACR_05) ont affirmé à propos du métro ne pas pouvoir emprunter des escaliers alors qu'elles habitaient en étage sans ascenseur. De même, le statut de l'espace du métro (intérieur ou extérieur) n'est pas clair et il semble qu'il cumule les difficultés des deux types d'espace. ACR_01 indique qu'elle n'utilise pas sa canne à l'intérieur, comme par exemple dans un centre commercial, mais qu'elle l'utiliserait si elle prenait le métro (ce qui serait donc une gêne $\left.-34^{\prime}\right)^{15}$. Pourtant, les espaces du métro sont perçus comme intérieurs lorsqu'il est question de regretter leur faible éclairage.

$\mathrm{Si}$ des actions physiques ou cognitives paraissent impossibles dans le métro et possibles dans un environnement fonctionnellement proche, c'est que d'autres raisons interviennent, en lien pourtant avec l'événement de santé. Quatre raisons ont été identifiées, d'ordre social ( $/ \mathrm{La}$ soumission au regard, spécificité de l'expérience en public », "L'inquiétude de ne pas trouver d'aide et l'inattention

\footnotetext{
15 Entre parenthèses sont indiqués les moments de l'entretien auxquels nous nous référons, pour les entretiens réalisés à Montréal.
} 
civile » « La reconfiguration de la demande de sociabilité au cours du vieillissement ») et géographique («L'attachement territorial et le sentiment d'étrangéité »).

\subsection{La soumission au regard, spécificité de l'expérience en public}

Les travaux d'Erving Goffman sont fondamentaux pour comprendre les ressorts des interactions qui adviennent en public. Celui-ci a comparé la situation à une scène dans le cadre de laquelle les acteurs interagissent sous le regard d'un public [28, chap.5]. Il a montré à quel point ce cadre rend lisible la situation pour les acteurs, qui savent comment se comporter, au risque d'être victimes d'une duperie, d'un cadre fabriqué dans le but de les tromper [28, p. 141]. Mais ce cadre est également ce qui rend la situation lisible pour les personnes extérieures, ce qui fait qu'un observateur extérieur est toujours en mesure de répondre à la question «que se passe-t-il ici ?» [28, p. 16].

I. Joseph a montré que le métro, en tant qu'espace public, était le support d'interactions sociales [not. 29]. S'il s'est surtout attaché à interroger à partir de ce cadre théorique la relation de service, c'est-à-dire les interactions entre voyageurs et agents, l'expérience du voyageur a été plus directement abordée par l'un de ses étudiants, Eloi Le Mouël [30], en préalable d'une thèse portant sur le vécu des œuvres culturelles dans les espaces normalement dédiés au déplacement. Il décrit par exemple, photos à l'appui, le cas d'une voyageuse dont le titre de transport ne fonctionne pas. Il s'agit probablement d'un abonnement car elle se refuse à le considérer comme invalide et à en acheter un autre. Si elle passe au-dessus du tripode de contrôle, il est probable que le public interprète son action comme une fraude, ce qui, pour une femme d'une quarantaine d'années, sera certainement perçu négativement. Elle doit donc donner à ce public les signes qui l'engagent à comprendre le cadre de la situation différemment : elle passe plusieurs fois de suite ostensiblement son titre de transport pour le valider, manifeste verbalement son incompréhension et sa contrariété, puis passe finalement par-dessus le tripode en continuant à grommeler. Elle évite ainsi de perdre la « face », c'est-à-dire d'être à la fois gênée et d'embarrasser les autres voyageurs. La « face » est le terme par lequel E. Goffman désigne la « valeur sociale positive qu'une personne revendique effectivement à travers une ligne d'action que les autres supposent qu'elle a adoptée au cours d'un contact particulier » [31, p. 9]. C'est quand l'acteur est pris dans une situation qui menace de lui faire perdre la « face », c'est-à-dire quand le regard du public devient menaçant que l'existence de ce regard devient lisible dans les paroles ou les conduites des autres voyageurs.

Il arrive que la personne soit porteuse d'un attribut qui lui fasse perdre la « face » sans que sa conduite ne soit en cause.
Erving Goffman parle alors d'un « stigmate » [32]. Cet attribut ne jette un discrédit sur la personne que dans le cadre d'une interaction avec d'autres en public. L'expérience d'ACR_03 témoigne du fait que la personne vieillissante, du fait de ses vulnérabilités physiques, est particulièrement porteuse d'attributs potentiellement stigmatisants : que ce soit la canne qu'elle utilise depuis une fracture du genou survenue six mois plus tôt (16') ou le simple fait de poser les deux pieds sur la même marche lorsqu'elle monte un escalier (28'), cette femme de 70 ans sait que sa conduite ne passe pas inaperçue. Mais ces attributs ne sont pas nécessairement porteurs de discrédit, au contraire : AU_02 en témoigne lorsqu'elle affirme : «Quand ils me voient avec mes cannes d'aveugle, les gens vont venir m'offrir de traverser la rue; ils vont venir voir si j'ai besoin de quelque chose ».

Parce qu'il n'existe que dans le cadre de relations, le stigmate dépend avant tout de la situation dans laquelle est engagée la personne : en l'occurrence, l'univers du métro constitue un cadre de situation spécifique. ACR_01 en témoigne : le fait de s'arrêter et de montrer une vulnérabilité est associé à l'inquiétude de gêner, « d'incommoder » les autres voyageurs.

(44'13) Enquêteur : Donc, vous vous êtes assise sur une marche et. . .

ACR_01 : Sur une marche et j'ai attendu que ça se replace un peu pour pouvoir poursuivre ma route. Ce qui fait que c'est un petit peu... c'est pour ça que j'ai décidé de modifier un peu... tandis que je n'ai pas cet inconvénient-là en prenant l'autobus.

D'accord. Parce que vous asseoir sur une marche et attendre... c'est trop compliqué pour vous ?... vous vous sentez...?

... c'est parce que là, j'arrête la circulation aussi.

Ah oui, d'accord.

Oui. Alors c'est tous les gens qui descendent, qui doivent se déplacer, eux autres aussi... alors que moi, je suis là assise et hop ! Ils arrivent à la course et tout d'un coup, ils font un saut et là, il y a une personne qui est assise là. Et puis, alors, ils sont obligés de me contourner et puis alors là, j'incommode les gens.

Tout se passe dans le métro comme si la personne était tenue d'une part à être aussi rapide que les autres voyageurs, d'autre part à se présenter au public comme compétente. Stéphane Tonnelat [33] a montré à partir du cas de New York que le métro constitue un système abstrait dont les experts référents ne sont que difficilement accessibles au public : la confiance dans ce système repose donc sur les voyageurs eux-mêmes. Cette confiance est d'autant plus importante que les espaces du métro n'offrent pas d'échappatoire aisée en cas de problème.

L'importance du regard du public est lisible lorsque les personnes concernées se demandent, comme PA_01, 85 ans, «Si je tombais, de quoi j'aurais l'air?». Dans cette courte 
phrase, le regard du public fait irruption pour expliquer un renoncement difficile à comprendre à partir de critères physiques : PA_01 avance en effet d'abord comme raison à son renoncement sa difficulté à monter et descendre les escaliers, ce qu'elle parvient pourtant à faire chez elles ou dans son supermarché, puis sa mauvaise vue, qui ne l'empêche pourtant pas de fréquenter les commerces situés dans le pôle du Pont de Sèvres sans jamais aller pour autant au-delà des tripodes de contrôle. Tous ces handicaps ne deviennent des freins à l'usage du métro que parce qu'ils se traduisent alors en stigmates.

La tendance à l'autodépréciation, que l'on retrouve de manière systématique dans les entretiens des personnes ayant renoncé à l'usage du métro est elle aussi un marqueur du rôle du regard du public. Les personnes interrogées se décrivent souvent comme "pas capables » (AR_01 par exemple, 11'30 et 15') ou " pas normales » (AU_03 : « je ne peux plus descendre normalement $\left.»-16^{\prime}\right)$. Il est à ce titre significatif de constater que les personnes plus jeunes qui renoncent à l'usage du métro sont au contraire beaucoup plus critiques envers les autres qu'envers elles-mêmes. JRP_03 a renoncé à l'usage du métro quitte à doubler son temps de déplacement en raison de la «promiscuité » subie dans ce mode ; de même que JRM_02, même si les déplacements et stationnements en voiture sont plus fluides à Montréal qu'à Paris. Les paroles de JRM_03, montréalaise de 30 ans, sont particulièrement explicites en ce qui concerne son aversion à l'égard des autres usagers (en l'occurrence non voyageurs) du métro.

(38') JRM_03 : ... Les tunnels ne sont pas toujours très propres non plus... [...]

Enquêteur : «Les tunnels ne sont pas très propres »... Qu'est-ce qui vous fait dire ça?

Ben c'est dans un mauvais état, il y a beaucoup de clochards dans les tunnels. Ce n'est pas forcément très bien aménagé parfois aussi... quoi d'autre. . . c'est assez sombre. [...]

Le fait qu'il y ait des clochards, ça change quelque chose pour vous? Par exemple, s'il y en a dans la rue, c'est différent que dans le métro ?

Non, c'est juste que dans le métro, ils t'approchent peut-être un petit peu plus souvent. Et aussi, certains d'entre eux, habitent dans ces tunnels-là donc ça... ça... Parfois, ils ont... ils utilisent les tunnels comme la maison, donc c'est vrai que ça pue parfois (rire)... parce qu'ils vont aux toilettes dans les... (rire)... quand ils ont un besoin... (silence)

D'accord. Et ça, dans la rue, c'est quelque chose qui...? Ben, ça se voit moins.

De fait, lorsque les plus jeunes renoncent à l'usage du métro, c'est bien plus fréquemment au profit de modes individuels (voiture, moto, vélo) que les personnes vieillissantes, qui lui préfèrent souvent le bus.

\subsection{L'inquiétude de ne pas trouver d'aide et l'inattention civile}

Les récits des personnes ayant renoncé au métro sont traversés par une constante : l'idée qu'elles ne trouveront pas d'aide en cas de nécessité. ACR_05 l'exprime explicitement : « Ou tu sais, d'un coup je me dis : "Si je tombe dans cet escalier-là et qu'il n'y a personne..." » (35'). PA_01 est aussi claire lorsqu'elle ajoute, après s'être demandée de quoi elle « aurait l'air» en cas de chute, « qui viendra [la] ramasser ». ACR_01 justifie sa réticence à demander de l'aide par le fait que les autres personnes lui semblent aller trop vite pour qu'elle s'autorise à les arrêter. Cette réticence n'est pas présente dans la rue ou dans l'autobus, et c'est ce qui fait le principal avantage de ce mode au regard du métro (AR_02-35').

L'analyse interactionniste des espaces publics a mis en évidence l'exigence, pour les acteurs engagés dans une situation, de rendre leur action lisible aux yeux d'un public. I. Joseph parle à ce titre d'une « hypertrophie de l'œil »[34, p. 43 ; 46]. Mais hypertrophie ne signifie pas omniprésence : un univers où nos faits et gestes seraient constamment visibles par un spectateur serait un univers concentrationnaire, à l'image du panoptique [35, pp. 233-234]. Les normes de l'action en public ménagent donc, en même temps que le devoir de lisibilité, un droit au retrait et à l'aparté. Une des règles qui structurent le cadre de l'expérience en public, l'inattention civile ${ }^{16}$, condense le droit de regard et le droit de retrait : «Elle consiste à montrer à autrui qu'on l'a bien vu et que l'on est attentif à sa présence (lui-même devant en faire autant) et, un instant plus tard, détourner l'attention pour lui faire comprendre qu'il n'est pas l'objet d'une curiosité ou d'une intention particulière. En faisant ce geste de courtoisie visuelle, le regard du premier peut croiser celui de l'autre, sans pour autant s'autoriser de « reconnaissance». Lorsque l'échange se déroule dans la rue, entre deux passants, l'inattention civile prend parfois la forme suivante : on jette un œil sur autrui à environ deux mètres de lui ; pendant ce temps on répartit par gestes les deux côtés de la rue, puis on baisse les yeux à son passage, comme pour une extinction des feux. C'est là, peut-être, le plus mineur des rituels interpersonnels, mais celui qui règle constamment nos échanges en société » $[36, \text { p. } 84]^{17}$. Loin d'être le symptôme d'une indifférence, elle est une marque de courtoisie, un soin porté à la co-présence.

Or, dans le métro, l'inattention civile prend des formes exagérées qui la font apparaître aux yeux de certains

\footnotetext{
${ }^{16} \mathrm{Ou}$ 《 inattention polie » selon la traduction proposée par Alain Kihm de l'expression civil inattention dans [59].

${ }^{17}$ Traduction proposée par I. Joseph à l'occasion de son article « Prises, réserves, épreuves » [60, p. 137].
} 
voyageurs comme une loi intangible [37]. Le détournement du regard, qui ne dure qu'un instant lorsque deux personnes se croisent dans la rue, se prolonge démesurément lorsque les personnes sont en co-présence dans les espaces confinés du métro, jusqu'à sembler imperturbable. La courtoisie disparait, et seule demeure l'indifférence. Il est pourtant connu, et les récits des personnes interrogées le confirment, qu'en cas de problème, par exemple si une personne paraît perdue ou chute, les autres voyageurs lui viendront en aide. JRP_01, jeune parisienne de 24 ans, raconte par exemple comment elle est venue en aide à un homme âgé qui avait glissé sur les sols humides de la station Saint-Lazare à Paris. Mais une fois en dehors de l'expérience du déplacement, le souvenir de l'atmosphère d'indifférence peut prendre le dessus et laisser penser aux personnes vieillissantes qu'elles n'y trouveront pas d'aide.

Or, les recherches portant sur le vieillissement $[38,39]^{18}$ ont montré qu'une perte d'autonomie fonctionnelle (physique ou cognitive) n'occasionnait pas nécessairement de dépendance précisément parce que la personne pouvait mettre en place des stratégies d'adaptation y compris en sollicitant d'autres personnes pour lui permettre de poursuivre ses activités comme précédemment. C'est d'ailleurs sur l'existence de ces stratégies d'adaptation que repose la distinction entre « déprise » et « désengagement ». La possibilité de demander de l'aide est fondamentale pour permettre à la personne de conserver son indépendance. Il est donc inévitable que les personnes vieillissantes affectées par une perte d'autonomie renoncent à prendre un mode de transport dans lequel elles ne sont pas assurées de trouver d'aide.

La comparaison entre personnes âgées ayant renoncé à l'usage du métro et personnes âgées toujours utilisatrices est à ce titre éclairante. Les propos de ces dernières montrent manifestement qu'elles n'hésitent pas à demander d'aide en cas de nécessité aux autres voyageurs ; c'est ce qui leur permet de prolonger leur usage du métro.

(16’30) Enquêteur : D'accord. Donc, si je récapitule, vous utilisez quatre moyens de transport : à pied, autobus, transport adapté et métro, c'est ça ?

AU_01 : Oui... Mais le métro, c'est. . . les gens disent : « Tu vas prendre le métro »... Mais on a toujours deux escaliers, à l'intérieur. On a un escalier mobile, ça c'est correct, il n'y

\footnotetext{
${ }^{18}$ Bernard Ennuyer a insisté sur la nécessité de faire une différence entre la dépendance comme incapacité à réaliser soi-même des actes fonctionnels de la vie courante et la perte d'autonomie qui désigne la privation de liberté de décider de son mode de vie par soi-même. Les incapacités à réaliser les principales activités de la vie quotidienne peuvent nécessiter, suivant le souhait de la personne, suivant son caractère, ses habitudes de vie et son environnement familial et géographique, une aide plus ou moins importante. La personne n'en reste pas moins libre de décider de son mode de vie, pour autant qu'elle n'a pas de désorientation d'ordre psychique importante.
}

a pas problèmes avec ma marchette ${ }^{19}$ mais les deux escaliers, j'ai toujours quelqu'un qui m'offre à descendre la marchette. C'est très rare que je vais descendre...Il y a deux paliers ordinairement huit marches par palier - c'est très rare que je n'ai pas quelqu'un qui va m'offrir de monter ou de descendre la marchette. Je l'accepte volontiers et puis, ils m'aident (rire)

Oui ? (rire)

Et il y en a... il y a des personnes ici qui disent : « Moi, je n'en serais pas capable ». Moi, j'ai déjà été 37 ans dans l'enseignement, j'ai d'jà assez aidé l'monde qu'ils peuvent m'aider! (rire)

« Je n'en serais pas capable », qu'est-ce que ça veut dire ?

Ben, ils n'iraient pas parce qu'ils n'accepteraient pas de se faire aider. Moi, je ne le demande pas. Des fois... je regarde (rire)... Surtout monter. Descendre : ça pourrait aller mais c'est quand-même. . Surtout quand je reviens avec des commissions, ma marchette, elle est plus pesante, plus lourde. J'ai une grosse marchette, ce n'est pas une petite que j'ai.

Donc vous n'avez pas besoin de demander mais des fois, vous demandez quand-même?

Je le demande par (mimant)... (rire)... et puis, je leur fais des compliments et les gens aiment ça ! (rire)...

Surtout quand c'est des beaux jeunes hommes...

... Ah bon! D'accord. .

...Ils aiment ça, que je leur fasse des compliments. J'ai appris ça (rire)...

\subsection{La reconfiguration de la demande de sociabilité au cours du vieillissement}

Les personnes vieillissantes ne se caractérisent pas uniquement par leurs vulnérabilités. Ce qui les distingue des autres personnes à mobilité réduite, c'est la reconfiguration sociale dans laquelle elles se trouvent engagées. La déprise se traduit par le double mouvement de disparition de liens anciens et de consolidation de certains nouveaux liens. Les causes de la disparition de liens sociaux anciens peuvent être multiples : départ en retraite, investissement des enfants dans d'autres cadres familiaux, décès de proches et veuvage. Elles se traduisent par un certain isolement de la personne sans que cela ne signifie pour autant solitude ou sentiment d'abandon : les adolescents semblent être la classe d'âge qui souffre de solitude plus que les personnes vieillissantes [40, p. 75]. Car la déprise est aussi un moment de redéploiement des liens sociaux soit par multiplication des liens dans l'espace public, soit par renforcement de certains liens avec des proches jusqu'alors plus distants. Ces deux éléments sont porteurs, à leur manière, de conséquences pour le choix modal des personnes vieillissantes.

\footnotetext{
19 Déambulateur.
} 
Les transports collectifs représentent un espace public à part entière et peuvent donc devenir un lieu de sociabilité pour les personnes vieillissantes. De moyens (de transport) pour atteindre une destination, ils peuvent même devenir destination en tant que telle. De ce fait, sont privilégiés les modes qui permettent cette sociabilité, avec les agents en présence comme avec les autres voyageurs. Le métro n'interdit pas cette forme de sociabilité, comme en témoigne AU_01, qui discute fréquemment avec des personnes dans le métro sans même avoir à engager elle la conversation (55' : «Ben ma fille, elle dit : "Je ne comprends pas ; tout le monde te parle !" (rire) »). Pour autant, la forme exagérée de l'inattention civile spécifique au métro et ses conséquences en termes d'atmosphère sociale de ce mode rendent le bus plus convivial et de ce fait plus attractif.

(19’30) ACR_02 ...Et puis, la semaine passée, j'ai pris un autobus qui était climatisé.

Enquêteur : Ah, oui?

Oui ! C'est nouveau ! Ils sont à l'essai ; il y en a deux. J'en ai pris un, il y a à peu près un mois qui était climatisé aussi. Dis donc : « Est-ce que je rêve ? » Je dis : « Avez-vous. . ? » - j'ai demandé au chauffeur (c'était une chauffeuse) - « Est-ce que je rêve ou vous avez l'air climatisé dans l'autobus ? »- elle me dit : « Vous ne rêvez pas !» [...]

Donc tu as échangé quelques mots avec la chauffeuse...

Ah, ben oui ! Moi, je leur dis toujours bonjour quand je rentre dans l'autobus.

... toujours bonjour?

Toujours. Moi, je leur dis bonjour - s'ils ne me le disent pas, c'est leur problème - et puis, quand je descends aussi : merci et puis bonjour, bonne journée.

Tu descends par l'avant, du coup ?

Oui. Souvent. S'il y a trop de monde, à l'heure de pointe, non... À l'heure de pointe, je vais sortir par l'arrière mais si ce n'est pas l'heure de pointe, comme ce matin pour m'en venir ici, je descends par l'avant. Il n'y a pas beaucoup de monde dans l'autobus, de toute façon, à ces heures-là. . . ce qui fait que je descends par l'avant et je dis merci, bonjour, bonne journée.

D'accord. « Merci, bonjour et bonne journée » et parfois, tu discutes un peu plus?

Oh ! Ça peut arriver s'il y a des personnes, des choses que... en général, non. Ils ne sont pas là pour jaser ; ils sont là pour faire leur travail. Mais s'il se passe quelque chose en avant, sur la route... il y a toujours des choses des fois, qui ne sont pas habituelles et à ce moment-là on peut (discuter).... si je suis à côté ! Mais, si je suis assise plus loin, je ne me lèverais pas pour aller parler au chauffeur.

Le renforcement de liens avec certains proches qui advient au cours de la déprise se traduit quant à lui par un report modal en défaveur des modes de transport collectif.
À Paris, et plus encore à Montréal, lorsque les proches accompagnent une personne vieillissante, cela se fait toujours en voiture. Aucune des personnes interrogées n'a d'ailleurs fait mention de la carte d'accompagnement dans les transports collectifs. Or, la demande d'accompagnement peut être, en même temps qu'une stratégie d'adaptation à une difficulté fonctionnelle, une façon de consolider des liens avec des proches. AR_02 explique ainsi, après avoir exposé les conséquences de la dégénérescence maculaire dont elle est affectée :

(10') AR_02 : Maintenant, je ne peux plus - à moins d'aller avec quelqu'un, je ne peux plus faire ça. Il me reste quelques activités. J'ai un neveu et une nièce qui s'occupent beaucoup, beaucoup de moi. Ils viennent régulièrement, ils s'occupent de ma lessive. Tous les samedis - là, aujourd'hui, je n'y suis pas allée parce que mon neveu n'était pas disponible, mais il y a un buffet d'art vocal, pas très loin d'ici, où on a un opéra tous les samedis après-midi, dans une petite salle qui contient 20-25 personnes. On nous sert un lunch et on nous donne un bel opéra sur écran alors ça je trouve ça bien, bien intéressant comme distraction. Et l'hiver... ça commençait aujourd'hui, justement, mais mon neveu, il était occupé, il ne pouvait pas venir aujourd'hui. Mais, autrement, il vient me chercher en auto. Hier, c'est ma nièce qui est venue me chercher en auto pour aller à la banque. Tu sais, j'ai beaucoup, beaucoup de services, d'attention des miens. Je n'ai plus de frères et sœurs - ils sont tous décédés - mais j'ai 22 neveux et nièces et j'ai juste à sonner une cloche pour qu'ils me rendent service, qu'ils me fassent des courses. Tout, à ce point de vue-là, je ne me plains pas d'être isolée ou de manquer du nécessaire.

(41'30 - fin de l'entretien, elle synthétise) : Non. Comme je vous dis, le métro, j'ai remplacé ça par... je vais aller un peu en autobus, de temps en temps, mais c'est rare. Je vais toujours avec mon neveu et ma nièce quand j'ai des sorties maintenant.

\subsection{L'attachement territorial et le sentiment d'étrangéité}

Au cours de notre travail de thèse [26], nous avons montré l'importance de l'attachement territorial dans un contexte d'espace de réseau pourtant consacré au passage et à la mobilité. À partir des récits des personnes interrogées, l'existence d'interactions entre les espaces du métro et des entités sociales de différents ordres a en effet été mise en évidence : ensemble des personnes en déplacement à un instant donné, ensemble des personnes habituées d'une station ou d'une ligne, groupe de personnes engagées dans une même revendication à l'égard de l'opérateur, etc. Du point de vue de la personne, ces interactions socio-spatiales 
se traduisent par des formes d'attachement à un espace dans un cadre collectif que nous avons qualifiées d'attachement territorial ${ }^{20}$. Notre travail doctoral a en particulier montré le rôle de cet attachement dans les déterminants de l'aisance du voyageur. L'aisance ne naît pas à proprement parler de l'attachement territorial, mais de la capacité du voyageur à alterner entre une perception allocentrée de l'espace du voyage dont le système signalétique constitue un rappel in situ et une perception égocentrée support de l'attachement territorial.

Les personnes vieillissantes ne font pas exception. Mais au cours du vieillissement, les difficultés physiques qu'elles rencontrent les obligent à adopter une perception égocentrée. À la suite d'un événement de santé, la personne est du jour au lendemain plongée dans la dimension la plus concrète de l'espace du réseau : couloirs à parcourir, escaliers à gravir, quais sur lesquels il faut attendre, voitures dans lesquelles on se déplace à proprement parler, etc. Or, cet espace est un espace qui résiste, qui n'est pas facile à parcourir ni même à percevoir : les espaces sont souvent les mêmes selon leur fonction (les couloirs se ressemblent, les espaces d'accueil sont tous aménagés selon le même modèle, les commerces sont de même marques) et les publicités qui habillent les murs changent trop souvent pour être support de l'orientation.

Nombreuses sont donc les personnes ayant renoncé au métro qui décrivent leurs dernières expériences de ce mode comme la traversée d'un espace confiné et contraignant. AR_01 explique ainsi la différence de la rue par rapport au métro : «On est plus libre. Dans le métro, on est enfermé, finalement » (23'30). Cette phrase est particulièrement intéressante parce qu'elle conclut un échange dans lequel cette vieille dame de 94 ans rend explicite le sentiment d'étrangéité qui est né non seulement à l'égard des espaces du métro, mais également à l'égard des autres personnes, et qui se traduit par l'abandon du métro, malgré la possibilité physique d'y accéder (hypothèse de la présence d'ascenseurs).

(22’30) AR_01 : J'ai arrêté de prendre le métro parce que je ne peux pas prendre le métro... (Mais) je pense qu'actuellement dans le métro, on a l'ascenseur maintenant, hein ?

Enquêteur : Euh... oui...

(Mais) non, je n'ai pas pris le métro depuis que j'ai ma chaise $^{21}$.

D'accord. Même s'il y a un ascenseur?

Même s'il y a un ascenseur. Non, je ne prendrais pas le métro.

\footnotetext{
${ }^{20}$ La notion de « territoire » est ici entendue dans son acception minimale d'interaction entre une entité sociale et une entité spatiale qui se développe dans le temps long.

${ }^{21}$ Fauteuil roulant.
}

Ah oui ?

Non. On rencontre toute sorte de monde dans le métro. C'est difficile des fois. [...] Je n'ai pas peur mais je m'aperçois qu'il y a toute sorte de monde qui prend le métro. C'est bien sûr, on rencontre toute sorte de monde.

C'est différent de la rue ?

Euh... On est plus libre, on dirait, dans la rue que dans le métro.

Si les personnes toujours utilisatrices ont tendance à assimiler la station la plus proche de leur domicile à un «chez nous », comme AU_06 qui explique « Rosemont ${ }^{22}$, tu sais, c'est comme si j'étais chez nous », les personnes qui y ont renoncé distinguent nettement l'espace du quartier de la station de métro. L'arrêt de bus se trouve « chez nous » mais la station de métro se trouve "près de chez nous ».

Il y a donc, en plus de la dimension sociale (évoquée en trois modalités), une dimension géographique des raisons du renoncement à l'usage du métro : les personnes qui renoncent à l'usage du métro témoignent d'un sentiment d'étrangéité, du sentiment de ne plus appartenir à un territoire, et ce, toujours en lien avec un événement de santé (la nécessité de se déplacer en « chaise » pour AR_01).

\section{Discussion : Le renoncement comme outil d'analyse du choix modal}

L'analyse du renoncement chez les personnes vieillissantes fait émerger des explications de leur comportement de déplacement qui apportent une contribution originale à la connaissance du choix modal, au-delà même de cette classe d'âge.

\subsection{Que sait-on du choix modal ?}

L'analyse du choix modal constitue un champ de recherche très actif qui tire son origine de la remise en cause de l'automobile issue des deux chocs pétroliers de 1973 et 1979 et de la prise de conscience des effets négatifs externes de ce mode (sécurité, environnement, conséquences sur le tissu urbain, etc.). L'enjeu principal de ce champ de recherche est donc de comprendre la préférence donnée à l'automobile. L'ouvrage de Vincent Kaufmann [41] propose une synthèse complète de ce champ à la fin des années 1990 ; les états de l'art de différents articles consacrés à cette question la complètent avec les travaux les plus récents [ $c f$. not. 42,43$]$.

Les premiers travaux ont fait émerger l'importance relative des avantages fonctionnels de l'automobile, en particulier en termes de temps (rapidité, disponibilité). Issus

\footnotetext{
${ }^{22}$ Station de métro de la branche est de la ligne Orange.
} 
d'une approche économique des transports, ils envisagent, pour les besoins de la modélisation, le choix modal comme relevant d'une décision économique effectuée par un individu. Des travaux complémentaires, relevant d'une approche géo-économique, montrent les liens fonctionnels qui unissent, dans une boucle de rétroaction, choix modal et organisation des territoires [44,45].

L'entrée de la sociologie et de la psychologie environnementale dans le champ de recherche couplée à une évolution de la géographie des transports vers une géographie sociale de la mobilité ont permis de compléter la liste des éléments expliquant la préférence généralisée pour l'automobile, jusqu'ici limitée aux paramètres de coût et de temps. L'avantage symbolique de ce mode est le plus précisément étudié tant son rôle est d'importance. Le choix de l'automobile est sous-tendu par les valeurs attribuées par chaque acteur en fonction de sa place dans le système social [46-48]. La voiture est porteuse de valeurs qui non seulement font défaut aux autres modes, mais sont en outre associées à l'utilisateur du véhicule beaucoup plus que dans le cas de modes collectifs ou non mécanisés et lui apportent un bénéfice symbolique direct : l'autonomie, la liberté, la puissance, etc. De plus, l'usage de l'automobile renvoie à l'appartenance à un groupe social qui peut être, selon les cas, celui des classes supérieures par opposition aux captifs [43] ou celui des locaux par opposition aux populations touristiques [49].

Des critères biaisant le mécanisme du choix lui-même ont été mis en évidence pour compléter l'analyse du choix modal : les biais perceptifs [50-52] et le rôle joué par l'habitude [53]. Le choix modal ne peut pas être un choix parfaitement rationnel dans la mesure où les usagers confrontent des paramètres qui sont déformés par des biais perceptifs propres aux situations de choix : une personne aura par exemple tendance à surestimer les temps de déplacement en transports collectifs si d'autres paramètres, non fonctionnels, la poussent à préférer la voiture. Elle aura également tendance à rechercher les informations qui la confortent dans son choix en ignorant celles qui pourraient le mettre en doute : elle n'est donc jamais en situation d'information parfaite, quelle que soit la disponibilité effective de cette information. La minimisation des efforts cognitifs nécessaires à la prise de décision tend à inscrire le choix modal dans l'habitude, la répétition d'un choix initial sans nouvelle confrontation des arguments, quelle que soit l'évolution effective de l'offre de transport. La formation de cette habitude revient en fait à annuler le choix en tant que tel.

Les résultats obtenus auprès des personnes âgées ayant modifié leurs habitudes modales en effectuant un transfert brutal ou progressif du mode métro vers d'autres modes permettent de contribuer à ce corpus de connaissances sur le choix modal sur quatre plans.

\subsection{Le choix par exclusion : une autre façon de concevoir le mécanisme décisionnel}

Le renoncement à un mode est une forme de décision en négatif, décision par exclusion de la possibilité jusqu'ici la plus adéquate au profit de celle qui occupait la seconde position. Cette forme de décision n'est pas envisagée puisque l'élaboration du concept de choix modal contient en germe l'idée que ce choix s'effectue en positif, en direction de l'automobile. Ceci explique que les efforts de la recherche se soient concentrés sur les avantages fonctionnels et symboliques de l'automobile, qui est conçue comme attirante, alors qu'elle peut représenter, notamment pour les « renonceurs ${ }^{23}$, un pis-aller à défaut d'autre mode ne serait-ce qu'en raison des coûts très élevés de possession et d'entretien d'une automobile. C'est alors du côté du mode préféré mais inaccessible qu'il faut aller regarder pour comprendre le choix modal.

Le fait que le choix modal puisse être un choix par exclusion et non uniquement un choix positif permet d'introduire l'idée que plusieurs mécanismes décisionnels peuvent correspondre au choix modal. Il invite à examiner ce qui relève de la décision, et à envisager le fait que celle-ci soit, plus qu'un résultat, un processus de formulation d'un problème et d'élaboration d'une réponse. L'intérêt porté à cet objet dans le cadre de la sociologie des organisations conduit également à imaginer les conséquences sur le choix modal du fait que celui-ci est pris dans le cadre d'un tissu de relations sociales, éventuellement constitué en organisation (par exemple dans le cas des Plans de déplacement d'entreprise). Les paroles des personnes vieillissantes témoignent de l'importance que jouent les relations aux proches dans le choix des modes empruntés : les nécessités d'accompagnement pour l'usage d'un mode peuvent être perçues comme une occasion de voir ses proches, ou au contraire être évitées pour conserver son autonomie. Au sein d'un ménage plus jeune mono-motorisé, l'usage de la voiture n'est jamais personnel non plus mais relève de négociations d'autant plus complexes qu'il y a d'enfants en âge de conduire.

\subsection{Le renoncement : rupture de l'habitude, moment de reconfiguration du système de valeurs}

L'habitude est, nous l'avons vu, un mécanisme de réduction de la charge cognitive affectée à la prise de décision, par alignement $\mathrm{du}$ choix présent sur la décision prise

\footnotetext{
${ }^{23}$ Mais également pour les personnes vivant dans des territoires dépendants à l'automobile.
} 
précédemment dans une situation conforme. Il semble que cette habitude soit suffisamment solide pour empêcher une reconfiguration du choix modal même lorsque l'offre de transport a été améliorée, par exemple même si une ligne de tramway vient d'être mise en place qui facilite le déplacement en transport collectif et réduit le nombre de voies affectées au transport individuel. Pourtant, le renoncement constitue une remise en cause de ces habitudes puissantes.

Le renoncement advient fréquemment à la suite d'un événement de santé ( $c f$. « résultats »). Il est probable que celui-ci joue un rôle en tant qu'événement de santé, parce qu'il modifie les capacités fonctionnelles de la personne, mais également en tant qu'événement de santé, modification du quotidien suffisamment forte pour rompre les habitudes et reconfigurer les choix modaux. L'événement de santé joue ainsi le même rôle pour les personnes âgées que d'autres événements, tels que la fin de l'année universitaire pour JRP_01, qui se trouve incapable, à son retour en Ile-de-France après trois mois de rupture, de recommencer à adopter une attitude agressive pour prendre le RER chaque matin aux Halles en direction de l'Université d'Orsay, et renonce à l'usage de ce mode au profit du vélo, ce qui l'oblige à changer d'université.

Le moment de reconfiguration du choix modal par renoncement à un mode est donc un moment de rupture de l'habitude suite à un événement, qui laisse la place à la prise en compte de nouveaux éléments qu'ils soient d'ordre fonctionnel ou symbolique. L'approche par le renoncement met en effet en évidence l'impermanence des systèmes de valeurs, qui varient notamment avec la place qu'occupe une personne dans un système social. L'entrée dans le grand âge par exemple, se traduit par un nouveau système de valeurs fréquemment rapporté au rythme : les plus jeunes semblent aller plus vite, la personne valorise un rythme calme, et a tendance à se sentir en inadéquation avec le rythme de la ville et des modes de transport collectifs en particulier.

\subsection{La mobilité \\ comme expression d'une expérience sociale située}

L'importance de l'approche par le renoncement pour la compréhension des comportements de mobilité en particulier en termes de choix modal met en évidence l'intérêt qu'il y a à connaître le vécu des voyageurs en situation, de manière générale et lors des événements qui ponctuent leur trajectoire modale. L'apport des chercheurs de deuxième génération sur le choix modal a été de penser la mobilité comme «l'expression d'une expérience sociale » [49], c'est-à-dire appréhendée en pratique et comme intrinsèquement inscrite dans un tissu social porteur de valeurs et de prescriptions de comportements. Il est indispensable aujourd'hui de lui adjoindre une dimension située, une connaissance de ce qui se joue lorsque le voyageur est en situation de déplacement.

La substitution de l'homo sociologicus à l'homo oeconomicus dans l'explication du choix modal s'est faite sur les fondements d'une théorie de l'action issue de la tradition wébérienne [41, p. 15, 49, 54]. Weber considère que l'action se comprend d'abord à l'échelle de l'individu, et que les raisons d'agir de cet individu peuvent être liées aux habitudes, aux affects et à un calcul économique, mais également aux valeurs qui lui sont propres [55]. Il semble nécessaire de donner à l'approche du choix modal une inflexion goffmanienne depuis longtemps mobilisée dans d'autres domaines de recherche sur la mobilité. Les « cadres » qui déterminent l'univers des possibles dans lequel les personnes décident de leurs actions ne sont pas uniquement les « champs » et les « habitus » qui leurs sont associés, pour reprendre la terminologie bourdieusienne [56]. Ce sont aussi les « cadres » de la situation ( $c f$. $\S$ « La soumission au regard, spécificité de l'expérience en public ») [28].

Envisager la dimension située de l'expérience sociale de la mobilité, c'est porter une attention accrue à la façon dont le voyageur entre en interaction avec les autres personnes qui fréquentent un espace de transit, mais également avec cet espace, et la façon dont cette interaction est porteuse d'aisance voire d'attachement à l'espace, ou au contraire de désorientation face à un espace qui résiste.

\subsection{Conséquences méthodologiques de l'approche située}

Les conséquences méthodologiques de cette proposition théorique sont l'importance renouvelée de l'approche qualitative, dont Per Gunnar Røe a déjà montré les intérêts pour la connaissance des comportements de mobilité [57], et surtout ici, l'importance du récit d'expérience en situation. Les raisons de choisir un mode de transport ne peuvent pas être comprises si l'expérience de la personne in situ n'est pas consignée en un récit. Les événements qui conduisent au renoncement ne peuvent en particulier être restitués que par le récit. Une personne ne décrira pas, lors d'un questionnaire, l'ensemble des difficultés ressenties suite à un événement de santé, mais s'en tiendra à un résultat («depuis mon opération de la hanche je ne peux plus descendre les escaliers », par exemple), et restreint ainsi l'analyse donc l'amélioration du réseau aux dimensions fonctionnelles de l'expérience du déplacement.

La méthode de l'entretien individuel semble particulièrement pertinente pour le recueil de l'expérience en situation. L'entretien peut être réalisé au domicile de la personne ou sous la forme de parcours commentés [58], qui se prêtent tout à fait aux enquêtes portant sur les déplacements, mais sont difficilement utilisables avec des personnes ayant 
complètement renoncé à un mode. La grille d'entretien doit se focaliser sur des questions pratiques, en particulier sur les événements qui conduisent au changement de mode : incident arrivé dans le mode ou premier déplacement dans ce mode après l'événement de santé considéré comme cause du changement de mode. L'enquête réalisée à Montréal nous a montré que les questions portant sur les situations d'interactions sociales ${ }^{24}$ ne donnent pas de réponse probante et qu'il est préférable d'attendre que la personne évoque ces situations d'interaction pour effectuer une relance sur le sujet. La grille ne doit donc pas chercher à aborder toutes les dimensions de l'expérience en situation, mais celles-ci doivent être bien listées dans le cadre des relances à effectuer. La grille doit être suffisamment longue toutefois pour que la personne ait le temps d'évoquer dans le détail ses expériences d'usage du mode, et de dépasser les raisons " objectives » (c'est-à-dire relevant de rationalités économiques ou de ce qui est communément admis comme une raison recevable) de choix modal avancées dans les premiers temps de l'entretien.

Comment l'opérateur qui souhaite améliorer son réseau de métro et limiter le renoncement à l'usage de ce mode peut-il s'assurer que les données qu'il tirera d'une série d'entretiens auprès de renonceurs seront suffisamment solides, pour ne pas dire représentatives, pour que les modifications qu'il engagera en conséquence soient efficaces? C'est toute la question de la valeur heuristique des méthodes qualitatives qui se pose, ici, à nouveau, cette fois de manière opérationnelle; deux réponses peuvent lui être apportées.

D'une part, les perceptions et représentations des personnes ne se laissent pas saisir de manière quantitative : lorsque les personnes doivent choisir dans un questionnaire les valeurs auxquelles elles associent tel ou tel mode de transport, elles vont choisir les valeurs derrière lesquelles elles mettent des définitions qui sont tout autres que celles imaginées par l'opérateur lorsqu'il a construit le questionnaire. C'est ainsi que les enquêtes sur la mobilité sont vérolées de notions "boîtes noires » telles que le « confort », la « praticité » ou, exemple archétypal de dissonance entre opérateur et usager, la « propreté $»^{25}$.

\footnotetext{
${ }^{24}$ Par exemple : Vous souvenez-nous d'une personne croisée dans le métro, d'un visage ? Vous souvenez-vous avoir discuté avec une personne dans le métro ? Vous souvenez-vous avoir fait une remarque à une personne dans le métro ? Vous souvenez-vous avoir signalé un problème auprès du personnel en station?

${ }^{25}$ Alors que l'opérateur pour qui est réalisée l'enquête entend, par le terme de «propreté » le degré de nettoyage des espaces et la présence ou non de détritus, les voyageurs eux se servent quasi-systématiquement de ce terme pour désigner pudiquement les personnes dont ils estiment la présence gênante. Un exemple tiré parmi une multiplicité d'autres, extrait d'un entretien réalisé à Montréal avec une femme de 30 ans ayant renoncé à l'usage du métro : (37'40) JRM_03 : « Les tunnels ne sont pas toujours très propres non plus... [...] Donc, je préfère 1'autobus au métro. » Enquêteur : « Les tunnels
}

D'autre part, lorsque des personnes décrivent une expérience socio-spatiale dans le détail que permet l'entretien, il est possible d'en déduire les cadres de son expérience, cadres qui engagent les autres personnes présentes. C'est la démarche de l'analyse proposée au chapitre trois : le récit d'une trentaine de personnes permet de comprendre certaines spécificités de l'environnement socio-spatial du métro, spécificités partagées entre les réseaux de Paris et Montréal. L'analyse qualitative est donc performante puisqu'elle permet de mettre au jour des principes qui concernent tout le monde quand l'analyse quantitative n'identifie que des constantes entre des parties d'une population.

Approches qualitatives et quantitatives ne sont pas pour autant opposées ni exclusives l'une de l'autre, mais sont des outils différents qui répondent à des questions différentes. Dans le cas du renoncement des personnes âgées, les méthodes quantitatives permettent d'envisager l'ampleur du phénomène, mais les raisons d'agir des personnes ne peuvent être envisagées en relation avec leur environnement socio-spatial que par l'analyse qualitative ; les mêmes conclusions peuvent être tirées pour les autres formes du choix modal.

\section{Conclusion}

L'apport, dans le champ de la mobilité, de l'approche relationnelle du vieillissement, à savoir le principe que la « déprise » engage la personne vieillissante dans un processus de reconfiguration de ses pratiques sociales et spatiales a été de mettre au jour l'existence d'un phénomène répandu de renoncement à l'usage des modes de ferrés lourds, et de lui apporter des explications qui complètent les premières raisons fonctionnelles les plus aisément identifiables, à savoir des raisons d'ordre social (dans l'interaction) et géographique. Ces résultats invitent à repenser l'analyse du choix modal pour l'ensemble des classes d'âge : avantage de l'entrée par le renoncement pour comprendre le processus décisionnel, dimensions situées (interactions sociales et spatiales) des raisons du choix modal, opérationnalité des méthodes qualitatives.

\section{Références bibliographiques}

1. Blanpain N, Chardon O (2010) Horizon 2060, un tiers de la population âgée de plus de 60 ans - Projection 2007-2060. Insee Première 1320.

2. Davey J (2007) Older people and transport: coping without a car. Ageing and Society 27: 49-65.

ne sont pas très propres. . Qu'est-ce qui vous fait dire ça? Vous avez une...? » JRM_03 : «Ben c'est dans un mauvais état, il y a beaucoup de clochards dans les tunnels. Ce n'est pas forcément très bien aménagé parfois aussi...». 
3. OCDE (2001) Vieillissement et transports. Concilier mobilité et sécurité. OCDE, Paris.

4. Joseph I (1987) Bus - Réseaux - Territoires. RATP, Département du Développement, Unité Prospective (rapport $n^{\circ} 20$ ), Paris.

5. Joseph I (1987) L'enjeu-station. La station de métro comme espace public. RATP, Département du développement, Unité prospective (rapport $\mathrm{n}^{\circ} 21$ ), Paris.

6. Dejeammes M, Livebardon C, Le Ruyet A, Benlahrech N (2001) La mobilité des personnes âgées : analyse des enquêtes ménages déplacements. CERTU, Lyon.

7. Kim S, Ulfarsson G (2004) Travel Mode Choice of the Elderly: Effects of Personal, Household, Neighborhood and Trip Characteristics. Journal of the Transp Res Board 1894: 117-126.

8. Rosenbloom S (2003) The Mobility Needs of Older Americans : Implications for Transportation Reauthorization. The Brookings Institution - Center on Urban and Metropolitan Policy, Washington DC, pp 1-20.

9. Secrétariat aux enquêtes O-D Métropolitaines (2008) Enquête Origine-Destination 2008. La mobilité des personnes dans la région de Montréal. Agence métropolitaine de transport (et al), Montréal.

10. Secrétariat aux enquêtes O-D Métropolitaines (2008) Enquête Origine-Destination 2008. Faits Saillants. Agence métropolitaine de transport (et al), Montréal.

11. STIF (2013) La mobilité des seniors. OMNIL - Fiche $n^{\circ} 19$ de l'Enquête globale transport 2009-11. Paris.

12. Bussière Y, Thouez J-P (2004) Mobility patterns of the disabled in Montreal in 1998 and long term perspectives. In : 10th International Conference on Mobility and Transport for Elderly and Disabled People, TRANSED 2004, 23-26 mai, Hamamatsu (Japan).

13. Dejoux V, Bussière YD, Madre J-L, Armoogum J (2010) Projection of the daily travel of ageing population: the Paris and Montreal Case, 1975-2020. Transp Rev $30: 495-515$.

14. Lord S, Joerin F, Thériault M (2009) Évolution des pratiques de mobilité dans la vieillesse : un suivi longitudinal auprès d'un groupe de banlieusards âgés. Cybergeo 8504 .

15. Pitaud P (2010) Solitude et isolement des personnes âgées. Eres, Toulouse.

16. Cumming E, Henry WE (1961) Growing Old. The Process of Disengagement. Basic Books, New York.

17. Barthe J-F, Clément S, Druhle M (1988) Vieillesse ou viellissement? Les processus d'organisation des modes de vie chez les personnes âgées. Les Cahiers de la Rech sur le Trav Soc 15 : $11-31$.

18. Clément S, Mantovani J (1999) Les déprises en fin de parcours de vie. Gérontologie et Société 90 : 95-108.

19. Clément S, Mantovani J, Membrado M (1996) Vivre la ville à la vieillesse. Se ménager et se risquer. Ann de la Rech Urbaine 73 : 91-98.

20. Comité sur l'accessibilité du métro de Montréal (2003) Rapport présenté au Ministre des Transports Mr Yvon Marcoux. Ministère des Transports du Québec, Québec.

21. Larrouy M (2011) L'invention de l'accessibilité. Des politiques de transports des personnes handicapées aux politiques d'accessibilité de 1975 à 2005. Presses Universitaires de Grenoble, Grenoble.

22. Amar G (1988) Pour une nouvelle conception des réseaux dans la ville. Quaderni $6: 23-33$.

23. Jeannot G, Joseph I, Amar G (1991) Services publics, l'espace de l'usager. RATP, Département du développement, Unité prospective (rapport $n^{\circ} 61$ ), Paris.

24. Joseph I (1999) Villes en gares. l'Aube, La Tour d'Aigues.

25. Clot-Goudard R, Tillous M (2008) L'espace du réseau : du flux au territoire. Le tournant pragmatiste engagé par Isaac Joseph. Tracés $15: 107-126$
26. Tillous M (2009) Le voyageur au sein des espaces de mobilité : un individu face à une machine ou un être socialisé en interaction avec un territoire? Les déterminants de l'aisance au cours du déplacement urbain. Thèse de l'Université Paris 1 Panthéon-Sorbonne, Géographie-Aménagement, sous la dir. Francis Beaucire, Paris.

27. Tillous M (2012) L'importance du lien dans le rapport au lieu. L'approche interactionniste comme façon de renouveler l'analyse du choix modal des personnes âgées. In : Espinasse C, Kaminagaï Y, Le Mouël E (eds), Lieux et Liens, actes du colloque de Cerisy du 26 mai au 2 juin 2009 (t. 2). 1'Harmattan, Paris, pp $67-86$.

28. Goffman E (1974) Les cadres de l'expérience. Minuit, Paris.

29. Joseph I (1988) Métro : espace public et relation de service. RATP, Département du Développement, Unité Prospective (rapport $\mathrm{n}^{\circ}$ 38), Paris.

30. Le Mouël E (2006) La culture dans les espaces non dédiés. Le cas de la RATP : les figures de l'usager citoyen et de l'usager client comme horizons de l'espace public. Thèse de l'Université Paris 10 - Nanterre, sous la dir. I Joseph et A Milon, Nanterre.

31. Goffman E (1967) Les rites d'interaction. Minuit, Paris.

32. Goffman E (1963) Stigmate : les usages sociaux des handicaps. Minuit, Paris.

33. Tonnelat S (2012) Confiance et émotions dans le métro de New York. In : Espinasse C, Kaminagaï Y, Le Mouël E (eds), Lieux et Liens, actes du colloque de Cerisy du 26 mai au 2 juin 2009 (t. 2). l'Harmattan, Paris, pp 201-228.

34. Joseph I (1984) Le passant considérable, essai sur la dispersion de l'espace public. Librairie des Méridiens, Paris.

35. Foucault M (2007 (1975)) Surveiller et punir : naissance de la prison. Gallimard, Paris.

36. Goffman E (1963) Behavior in Public Places: Notes on the Social Organization of Gatherings. Free Press of Glencoe, New York.

37. Levine J, Vinson A, Wood D (1973) Subway Behavior. In : Birenbaum A, Sagarin E (eds). People in places: The sociology of the familiar. Praeger, New York/Washington, pp 208-216.

38. Ennuyer B (2004) Les malentendus de la dépendance : de l'incapacité au lien social. Dunod, Paris.

39. Ennuyer B (2007) 1962-2007: Regards sur les politiques du maintien à domicile et sur la notion de libre choix de son mode de vie. Gérontologie et Société 123.

40. Caradec V (2001) Sociologie de la vieillesse et du vieillissement. Armand Colin, Paris.

41. Kaufmann V (2000) Mobilité quotidienne et dynamiques urbaines : la question du report modal. Presses Polytechniques et Universitaires Romandes, Lausanne.

42. Beirão G, Sarsfield Cabral JA (2007) Understanding Attitudes Towards Public Transport and Private Car: a Qualitative Study. Transp Policy 14: 478-489.

43. Brisbois X, Kouabenan DR, Rubens L (2012) Mixité sociale et choix modal : importance des dimensions symboliques dans l'attrait des transports collectifs. Recherche Transports Sécurité 28: 181-189.

44. Merlin P (1984) La planification des transports urbains. Masson, Paris.

45. Dupuy G (1995) Les territoires de l'automobile. Anthropos/Economica, Paris.

46. Anable J (2005) "Complacent Car Addicts" or "Aspiring Environmentalists"? Identifying travel behaviour segments using attitude theory. Transp Policy 12: 65-78.

47. Steg L (2005) Car use: lust and must. Instrumental, symbolic and affective motives for car use. Transp Res Part A Policy Pract 39:147-162.

48. Gärling T, Axhausen K, Brydsten M (1996) Travel choice and the Goal/Process Utility Distinction. Appl Cogn Psychol 10: 65-74. 
49. Petit J (2002) La mobilité comme figure de l'expérience sociale : conséquences sur la caractérisation de la demande de transport. Recherche Transports Sécurité $76: 190-207$.

50. O'Farrel P, Markham J (1974) Commuter perception of public transport work journey. Environ Plan A 6: 79-200.

51. Bailly A (1979) La perception des transports en commun par l'usager. Transp Environ Circ 32: 23-28.

52. Flamm M (2003) Comprendre le choix modal. Les déterminants des pratiques modales et des représentations individuelles des moyens de transport. Thèse de l'Ecole Polytechnique Fédérale de Lausanne, Faculté de l'Environnement Naturel, Architectural et Construit (ENAC), sous la dir. Michel Bassand, Lausanne.

53. Goodwin P (1985) Évolution de la motivation des usagers en matière de choix modal. In : Table ronde $\mathrm{n}^{\circ} 68$. Conférence européenne des ministres des transports, Paris.
54. Petit J (2003) Cinq logiques de mobilité et leurs conséquences sur la planification des déplacements urbains. Cah Sci du Transp 43 : $35-58$.

55. Weber M (1922) Économie et société, Plon, Paris.

56. Bourdieu P (1980) Le sens pratique, Minuit, Paris.

57. Røe PG (2000) Qualitative Research on intra-urban travel: an alternative approach. Journal of Transp Geogr 8: 99-106.

58. Thibaud J-P (2001) La méthode des parcours commentés In : Grosjean M, Thibaud J-P (eds.), L'espace urbain en méthodes, Parenthèses, Marseille, pp. 79-100.

59. Goffman E (1959) La mise en scène de la vie quotidienne. t.2 Les relations en public, Minuit, Paris.

60. Joseph I (1997) Prises, réserves, épreuves. Communications 65 : 131-142. 
Annexe 1. Profil détaillé des personnes interrogées

Paris

Personne de 60 ans et plus (4)

PA_01

85 ans

PA_02

60 ans

PA_03

69 ans

PA_04

76 ans

Personnes de moins de 60 ans ayant renoncé au métro (3)

JRP_01

24 ans

JRP_02

26 ans

JRP_03

55 ans

Montréal

Personne de plus de 65 ans ayant renoncé au métro (2)

AR_01

94 ans

AR_02

Personne de plus de 65 ans en cours de renoncement (renoncement « de fait ») (5)

ACR_01

ACR_02

ACR_03

ACR 04

ACR_05

78 ans

Personnes de plus de 65 ans toujours utilisatrices (9)

AU_01

80 ans

AU_02

78 ans

AU_03

83 ans

AU_04

71 ans

AU_05

80 ans

AU_06

79 ans

AU_07

85 ans

AU_08

78 ans

AU_09

86 ans

Personnes de moins de 50 ans ayant renoncé au métro (4)

51 ans

JRM_01

38 ans

JRM_02

30 ans

JRM_03

48 ans

JRM_04 